\title{
Estrutura e sucessão ecológica de uma comunidade florestal urbana no sul do Espírito Santo
}

Structure and ecological succession of an urban forest community

in the south of the Espírito Santo

\author{
Wiane Meloni Silva ${ }^{1}$, João Paulo Fernandes Zorzanelli ${ }^{2,5}$, Julia Siqueira Moreau ${ }^{3}$, \\ Karla Maria Pedra de Abreu ${ }^{4} \&$ Sustanis Horn Kunz ${ }^{3}$
}

\begin{abstract}
Resumo
Comunidades florestais são ambientes dinâmicos, nas quais processos de sucessão ocorrem naturalmente, podendo conduzir à maturidade da floresta. $\mathrm{O}$ objetivo deste estudo foi avaliar a estrutura do componente arbustivo-arbóreo de um fragmento de Floresta Estacional Semidecidual e caracterizar o seu estágio sucessional. O estudo foi realizado no município de Alegre, Espírito Santo, na ARIE Laerth Paiva Gama. A amostragem de todos os indivíduos com DAP $\geq 5 \mathrm{~cm}$ foi realizada por meio de pontos-quadrantes. Além dos parâmetros ecológicos foi obtida a estrutura diamétrica para a comunidade. As espécies foram classificadas quanto ao grupo ecológico em pioneiras, secundárias iniciais e secundárias tardias, e quanto ao grau de ameaça de extinção. Foram registradas 112 espécies, 81 gêneros e 34 famílias, das quais se destacaram em Valor de Importância Pseudopiptadenia contorta (15,33), Apuleia leiocarpa (5,08), Acosmium lentiscifolium $(4,42)$ e Parapiptadenia pterosperma $(4,30)$. O índice de Shannon foi de 4,17 e a equabilidade de 0,87 , sendo considerados valores elevados para florestas estacionais semideciduais. Com relação à avaliação dos grupamentos ecológicos, as secundárias tardias e iniciais se destacaram em riqueza, no entanto as iniciais foram mais abundantes na comunidade. Deste modo, o remanescente florestal estudado pode ser caracterizado como pertencente ao estágio sucessional inicial-intermediário.
\end{abstract}

Palavras-chave: espécies ameaçadas, fitossociologia, floresta estacional semidecidual, Unidade de Conservação.

\begin{abstract}
Forest communities are dynamic environments in which succession processes occur naturally, may lead to the maturity of the forest. The objective of this study was to evaluate the structure of the woody component of a fragment of semideciduous forest and characterize their successional stage. The study was conducted in the municipality of Alegre, Espirito Santo, in ARIE Laerth Paiva Gama. The sampling of all individuals with $\mathrm{DBH} \geq 5 \mathrm{~cm}$ was performed by points quadrants. In addition to the ecological parameters it was obtained diameter structure to the community. The species were classified according to environmental group in pioneers, early secondary and late secondary, and the degree of threat of extinction. Were recorded the 112 species, 81 genera and 34 families, of which have excelled in importance value were Pseudopiptadenia contorta (15.33), Apuleia leiocarpa (5.08), Acosmium lentiscifolium (4.42) and Parapiptadenia pterosperma (4.30). The Shannon index was 4.17 and the evenness of 0.87 and are considered high values for semideciduous forests. As to the evaluation of ecological groups, late and early secondary stood out in wealth, however initials were more abundant in the community. Thus, the remaining forest studied can be characterized as belonging to the initial-intermediate stage succession.
\end{abstract}

Key words: endangered species, phytosociology, semi-deciduous forest, Conservation Unit.

\footnotetext{
${ }^{1}$ Universidade Federal de Viçosa, Prog. Pós-Graduação em Ciência Florestal, Av. Peter Henry Rolfs s/n, Campus Universitário, 36570-900, Viçosa, MG, Brasil.

${ }^{2}$ Universidade Federal do Espírito Santo, Prog. Pós-Graduação em Ciências Florestais, Av. Governador Lindemberg 316, 29550-000, Jerônimo Monteiro, ES, Brasil.

${ }^{3}$ Universidade Federal do Espírito Santo (UFES), Depto. Ciências Florestais e da Madeira, Av. Governador Lindemberg 316, 29550-000, Jerônimo Monteiro, ES, Brasil.

${ }^{4}$ Instituto Federal de Educação, Ciência e Tecnologia do Espírito Santo, Campus Alegre, Rodovia 482 Km 47, 29520-000, Alegre, ES, Brasil.

${ }^{5}$ Autor para correspondência: jp.zorzanelli@gmail.com
} 


\section{Introdução}

A Floresta Atlântica é um complexo de fisionomias vegetacionais, como as florestas ombrófilas densas, florestas ombrófilas mistas, florestas estacionais deciduais e semideciduais (Joly et al. 2014), que se estende do sul ao nordeste do Brasil, além de se projetar aos territórios do Paraguai e da Argentina (Tabarelli et al. 2010). Devido aos fortes impactos humanos, como expansão dos cultivos agrícolas, processos de industrialização e formação dos grandes núcleos urbanos caracterizando uma paisagem extremamente fragmentada, e por ser um domínio contendo elevada diversidade de plantas e animais, inclusive altos níveis de endemismo (Forzza et al. 2012; Scarano \& Ceotto 2015). Myers et al. (2000) consideraram esse bioma como um dos grandes hotspots mundiais prioritários para conservação.

A fragmentação da Floresta Atlântica tem sido amplamente discutida (Rodrigues \& Nascimento 2006; Metzger et al. 2009; Colombo \& Joly 2010; Joly et al. 2014; Pereira et al. 2015), consistindo uma das grandes ameaças à biodiversidade, resultando na perda da variabilidade genética e na extinção de espécies. Pardini et al. (2009), Ribeiro et al. (2009) e Tabarelli et al. (2010) indicaram o planejamento da gestão de "paisagens modificadas pela ação antrópica" (Tabarelli et al. 2010) e a conectividade de pequenos fragmentos florestais, comuns na paisagem da Floresta Atlântica (Matte et al. 2015), como forma de conservar espécies e manter os serviços ambientais. Um dos trabalhos básicos para esse planejamento fundamenta-se na caracterização florístico-estrutural desses remanescentes.

$\mathrm{Na}$ região sudeste do Brasil, estudos da estrutura e composição florística de florestas estacionais semideciduais são comuns (por exemplo, Souza et al. 2012; Moreira et al. 2013; Souza et al. 2013; Gris et al. 2014; Rezende et al. 2015), cujos esforços geraram valiosas informações para pesquisas mais complexas como a evolução e ecologia de florestas estacionais (Pennington et al. 2009), além de proverem dados ao estudo dos processos e mecanismos ecológicos que envolvem a dinâmica das espécies em relação a variáveis ambientais e sucessão ecológica, fornecendo instrumentos à gestão e proteção de recursos naturais para as Unidades de Conservação.

Entretanto, a caracterização florísticaestrutural das comunidades florestais típicas da Floresta Estacional Semidecidual no estado do
Espírito Santo é pouco difundida, especialmente na região sul onde a fisionomia se destaca nas regiões de baixas altitudes. Os trabalhos realizados no seu âmbito estão concentrados no corredor ecológico Burarama-Pacotuba-Cafundó (Araújo et al. 2015) documentados por Archanjo et al. (2012) e Abreu et al. (2013).

Na Floresta Nacional (FLONA) de Pacotuba, Abreu et al. (2013) avaliaram a estrutura da comunidade arbórea e concluíram que o fragmento se encontra em estágio intermediário de sucessão. Entretanto, os autores destacaram a expressiva riqueza, a presença de espécies de importância conservacionista e a necessidade dessa área ser priorizada em programas de manejo e conservação na região.

Archanjo et al. (2012) avaliaram a estrutura horizontal do componente arbóreo da Reserva Particular do Patrimônio Natural (RPPN) Cafundó, e relataram que o remanescente florestal é composto predominantemente por espécies secundárias tardias, caracterizando-se como um fragmento bem conservado e que detém alta diversidade de espécies contendo flora arbórea peculiar.

Outra ferramenta importante para o planejamento e gestão das áreas protegidas é a avaliação da sucessão vegetal, possibilitando melhorar as técnicas de manejo da vegetação e gerenciamento dos recursos naturais. Prach \& Walker (2011) elencaram que a caracterização dos processos de sucessão também é igualmente importante para prover informações sobre a perda da biodiversidade, mudanças climáticas, invasão de espécies e restauração ecológica. Grande parte dos estudos de sucessão ecológica, têm utilizado os métodos de avaliação dos grupos funcionais (Gandolfi et al. 1995) para predizerem o nível de conservação de determinado remanescente florestal, embora ainda sejam um recurso limitado para caracterizar a real situação do mesmo.

Como forma de fornecer ferramentas para a gerência de uma Unidade de Conservação, a Área de Relevante Interesse Ecológico (ARIE) Laerth Paiva Gama, no município de Alegre, Espírito Santo, constituíram objetivos deste trabalho, caracterizar a estrutura da comunidade arbustivoarbórea de um trecho de Floresta Estacional Semidecidual e seu estágio sucessional, atendendo às seguintes hipóteses: (1) a comunidade estudada se encontra com estrutura fitossociológica similar a florestas estacionais conservadas e (2) a vegetação pertence ao estágio médio de regeneração. 


\section{Material e Métodos}

\section{Área de estudo}

Este estudo foi realizado na ARIE Laerth Paiva Gama, inserida na bacia do rio Alegre, município de Alegre, Espírito Santo. A área protegida em questão localiza-se junto ao perímetro urbano e possui aproximadamente 27,57 ha, estando situada nas coordenadas geográficas $20^{\circ} 46^{\prime} 03,44^{\prime \prime} \mathrm{S}, 41^{\circ} 32^{\prime} 57,24^{\prime \prime} \mathrm{W}$, com altitude aproximada de $250 \mathrm{~m}$.

A localidade, em tempos pretéritos, experimentou diversas alterações em virtude das ações antrópicas para expansão de áreas agricultáveis e, em 1992, foram realizados plantios de reflorestamento com espécies nativas e exóticas, resultando em duas matrizes florestais. Esses plantios correspondem ao reflorestamento de eucalipto (Corymbia citriodora (Hook.) K.D. Hill \& L.A.S. Johnson) e acácia (Acacia auriculiformis Benth. e Acacia mangium Willd) (3,87 ha) e uma área de floresta mista (3,26 ha) onde foi implantado um reflorestamento com diversas espécies nativas e exóticas (Fig. 1). O fragmento de floresta nativa onde o estudo foi realizado, situa-se no trecho superior da ARIE e possui aproximadamente 18,81 ha. Atualmente é importante ressaltar que existem comunidades no entorno dessa área, as quais usam a ARIE como acesso, o que pode causar dano e dificuldade à sua preservação.

O clima da localidade, segundo a adaptação de Köppen para o Brasil (Alvares et al. 2013), é do tipo Aw tropical úmido, caracterizado por inverno seco e verão chuvoso, com temperaturas médias anuais de $23^{\circ} \mathrm{C}$ e o volume de precipitação superior a $1400 \mathrm{~mm}$ por ano, concentrado especialmente no verão. A vegetação pertence ao domínio da Floresta Atlântica e está inserida na fitofísionomia da Floresta Estacional Semidecidual (Ribeiro et al. 2009; Joly et al. 2014). O solo predominante é classificado como Latossolo Vermelho-Amarelo (Espírito Santo 1994).

\section{Métodos}

A amostragem da estrutura da comunidade arbustivo-arbórea foi realizada por meio do método de pontos-quadrantes (Durigan 2003), sendo
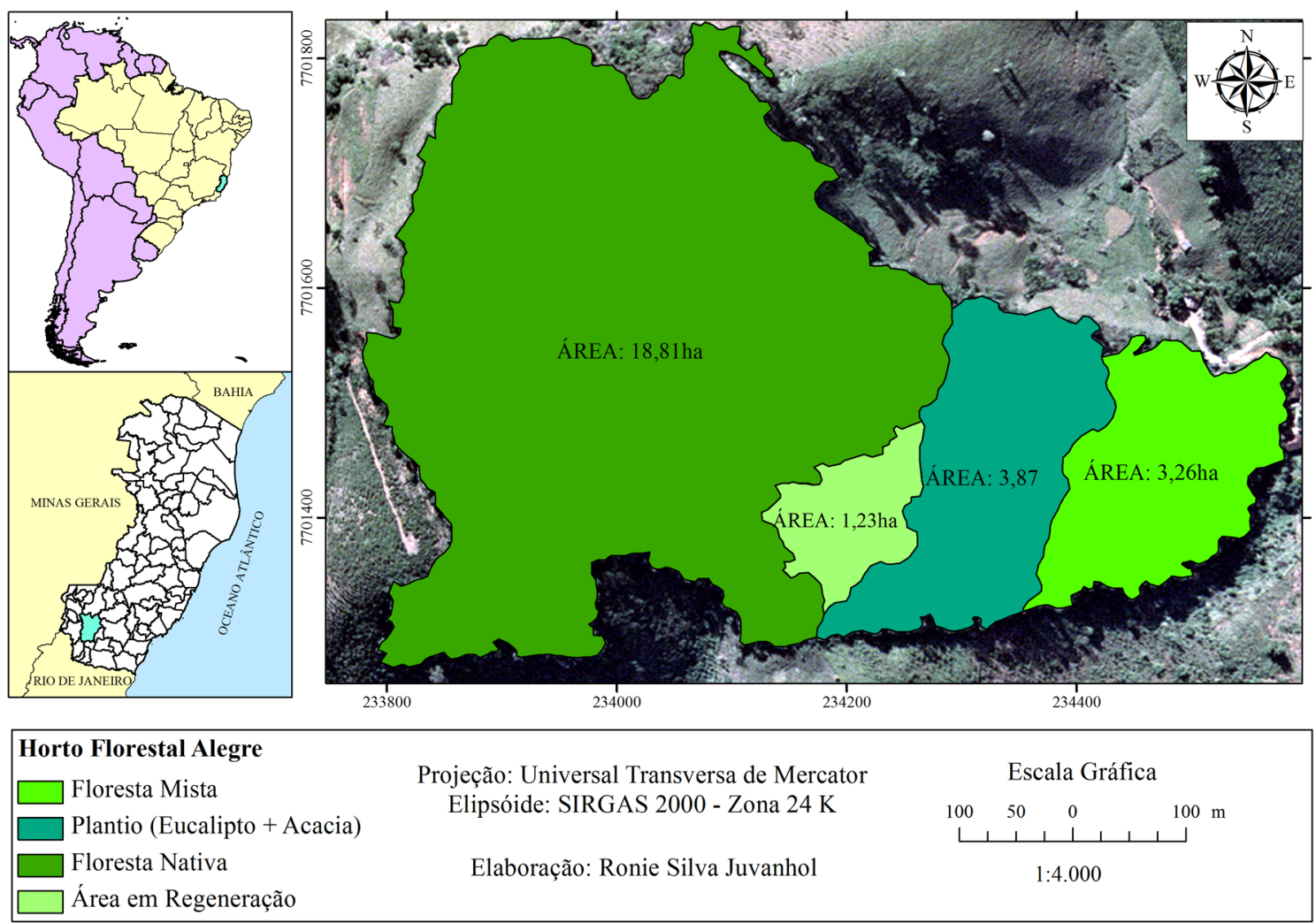

Projeção: Universal Transversa de Mercator Elipsóide: SIRGAS 2000 - Zona $24 \mathrm{~K}$

Elaboração: Ronie Silva Juvanhol

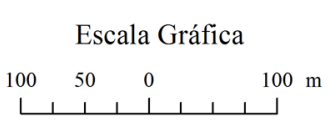

$1: 4.000$

Figura 1 - Localização da área de estudo, município de Alegre, Espírito Santo. Juvanhol (2013).

Figure 1 - Location of the study area, municipality of Alegre, Espirito Santo. Juvanhol (2013). 
avaliadas 100 unidades amostrais, distribuídas em sete linhas distanciadas entre si por $30 \mathrm{~m}$. Os pontos-quadrantes foram dispostos $20 \mathrm{~m}$ um do outro para evitar sobreposição dos indivíduos amostrados, abrangendo toda a área de estudo e evitando o efeito de borda. Foram inventariados os quatro indivíduos mais próximos do centro da cruzeta, um para cada quadrante. Em seguida, foi mensurado o diâmetro a altura do peito (DAP) maior ou igual a $5 \mathrm{~cm}$ para todos os indivíduos, além da distância da árvore ao centro da cruzeta. Plantas perfilhadas tiveram todos os perfilhos medidos, desde que pelo menos um deles atendesse ao critério de inclusão adotado.

Foram coletados os materiais botânicos reprodutivos e/ou vegetativos de todos os indivíduos amostrados. A identificação foi realizada por comparação com exsicatas do herbário VIES Setorial de Jerônimo Monteiro, sinopses e revisões taxonômicas, além de material bibliográfico atual. Os materiais férteis foram depositados no herbário VIES Setorial de Jerônimo Monteiro. As famílias foram classificadas com base em APG IV (Angiosperm Phylogeny Group 2016) e grafia e sinonímias das espécies, inclusive a autoria, foram averiguadas por meio do banco de dados online da Flora do Brasil 2020 em construção (2016). Foi verificada no Livro Vermelho da Flora do Brasil (Martinelli \& Moraes 2013) a existência de espécies ameaçadas de extinção.

A estrutura horizontal e a diversidade florística foram avaliadas através dos descritores ecológicos (densidade, dominância e frequência relativas e valor de importância (VI)) e do índice de diversidade de Shannon (H') e equabilidade de Pielou (J), respectivamente, seguindo-se Brower et al. (1998). Esses dados foram analisados por meio do software Fitopac 2.1. (Shepherd 2009).

Para caracterização dos grupos ecológicos, as espécies foram classificadas nas categorias sucessionais como pioneiras (PI), secundárias iniciais (SI) e secundárias tardias (ST), segundo a classificação proposta por Budowski (1965), fundamentado pelos trabalhos de Fonseca \& Rodrigues (2000), Santos et al. (2004), Silva et al. (2004), Marangon et al. (2007), Carvalho et al. (2007), Braga et al. (2011), Lopes et al. (2011). As espécies com ausência de informações ecofisiológicas permaneceram classificadas como "não caracterizadas" (NC).

A estrutura diamétrica foi obtida para auxiliar na detecção do estágio sucessional da vegetação. Assim, as classes de diâmetro e os intervalos de classe foram estimados com base na equação de Spiegel (1976), sendo utilizado o menor DAP como limite inferior da primeira classe diamétrica. Esse mesmo parâmetro foi avaliado também para as cinco espécies com maior VI.

\section{Resultados e Discussão}

Foram amostrados 399 indivíduos na ARIE Laerth Paiva Gama, sendo representados por 112 espécies, pertencentes a 81 gêneros e 34 famílias botânicas. Fabaceae (22 espécies), Meliaceae (11), Sapotaceae (10), Anacardiaceae, Nyctaginaceae e Euphorbiaceae (três cada) foram as seis famílias botânicas com maior riqueza específica, correspondendo a $50 \%$ do total de espécies. Dos indivíduos amostrados, 76\% foram identificados em nível de espécie, 19,5\% permaneceram apenas com nome genérico, 3,5\% em nível de família e $1 \%$ compuseram os indivíduos não identificados.

A respeito da estrutura fitossociológica, as dez espécies de maior Valor de Importância (VI) foram Pseudopiptadenia contorta, Apuleia leiocarpa, Acosmium lentiscifolium, Parapiptadenia pterosperma, Piptadenia gonoacantha, Senegalia polyphylla, Trichilia pallida, Guapira opposita, Trichilia casaretti e Siparuna guianensis, as quais compuseram 45,9\% do VI total.

As três primeiras espécies com maior VI destacaram-se devido às densidades e dominâncias elevadas na área de estudo, sendo $P$. contorta aquela com os maiores valores para todos os parâmetros estimados. T. pallida apresentou indivíduos com menor diâmetro, entretanto com elevada densidade se comparada aos indivíduos de $P$. pterosperma, P. gonoacantha e Senegalia polyphylla. Devido a características ecofisiológicas e do ambiente de distribuição das próprias espécies, algumas naturalmente não atingem grandes diâmetros (Imaña-Encinas et al. 2008), como pode ser verificado para $T$. pallida. Algumas espécies do gênero Trichilia são relatadas como de sub-bosque, ocorrendo como abundantes e apresentando menor altura e menor área basal, ocupando estrato inferior e médio da floresta (Sevilha et al. 2001; Bianchini et al. 2010; Almeida et al. 2015).

Apesar disso, a importância ecológica de $T$. pallida foi influenciada por sua baixa dominância. Siparuna guianensis apresentou maior número de indivíduos que $S$. polyphylla, $P$. pterosperma e G. opposita, mas esteve pouco distribuída ao longo da área, ou seja, possui distribuição mais restrita apresentando possível padrão de agregação $(\mathrm{FR}=2,89 \%)$. 
Tabela 1 - Espécies encontradas na amostragem do fragmento florestal da ARIE Laerth Paiva Gama, Alegre, Espírito Santo, ES e seus respectivos parâmetros fitossociológicos estimados - NI. número de indivíduos; DR. densidade relativa (\%); FR. frequência relativa (\%); DoR. dominância relativa (\%); VI. valor de importância relativo (\%); GE. grupo ecológico; PI. pioneira; SI. secundária inicial; ST. secundária tardia; NC. não caracterizada.

Table 1 - Species founded in the sampling of forest fragment of ARIE Laerth Paiva Gama, Alegre, Espirito Santo, and their respective estimated phytosociology parameter - NI. number of individuals; DR. relative density (\%); FR. relative frequency (\%); DoR. relative dominance (\%); VI. importance value (\%); GE. ecological group; PI. pioneer; SI. initial secondary; ST. late secondary; NC. not characterized.

\begin{tabular}{|c|c|c|c|c|c|c|}
\hline Espécies & NI & DR & FR & DoR & VI & GE \\
\hline Pseudopiptadenia contorta (DC.) G.P. Lewis \& M.P. Lima & 47 & 11,78 & 9,28 & 24,99 & 46,05 & PI \\
\hline Apuleia leiocarpa (Vogel) J.F. Macbr. & 16 & 4,01 & 4,35 & 6,92 & 15,28 & SI \\
\hline Acosmium lentiscifolium Schott & 18 & 4,51 & 4,06 & 4,71 & 13,28 & ST \\
\hline Parapiptadenia pterosperma (Benth.) Brenan & 12 & 3,01 & 3,19 & 6,73 & 12,93 & SI \\
\hline Piptadenia gonoacantha (Mart.) & 8 & 2,01 & 2,03 & 5,68 & 9,71 & SI \\
\hline Senegalia polyphylla (DC.) Britton \& Rose & 13 & 3,26 & 3,48 & 2,45 & 9,18 & SI \\
\hline Trichilia pallida Sw. & 16 & 4,01 & 2,9 & 1,54 & 8,45 & ST \\
\hline Guapira opposita (Vell.) Reitz & 11 & 2,76 & 3,19 & 2,17 & 8,11 & SI \\
\hline Trichilia casaretti C.DC. & 10 & 2,51 & 2,9 & 2,05 & 7,46 & ST \\
\hline Siparuna guianensis Aubl. & 14 & 3,51 & 2,9 & 1 & 7,41 & SI \\
\hline Astronium graveolens Jacq. & 9 & 2,26 & 2,61 & 1,85 & 6,72 & SI \\
\hline Astronium conccinum Schott. & 10 & 2,51 & 2,61 & 1,43 & 6,54 & SI \\
\hline Senefeldera verticillata (Vell.) Croizat & 8 & 2,01 & 1,74 & 1,57 & 5,32 & ST \\
\hline Machaerium nyctitans (Vell.) Benth. & 5 & 1,25 & 0,87 & 2,94 & 5,06 & PI \\
\hline Actinostemon verticillatus (Klotzsch) Baill. & 8 & 2,01 & 2,03 & 0,56 & 4,59 & ST \\
\hline Trichilia silvatica C.DC. & 7 & 1,75 & 2,03 & 0,56 & 4,34 & ST \\
\hline Casearia sylvestris $\mathrm{Sw}$. & 6 & 1,5 & 1,74 & 0,86 & 4,1 & SI \\
\hline Neoraputia alba (Ness \& Mart.) Emmerick ex Kallunki & 7 & 1,75 & 1,45 & 0,88 & 4,08 & ST \\
\hline Copaifera lucens Dwyer & 4 & 1 & 1,16 & 1,81 & 3,97 & SI \\
\hline Sorocea guilleminiana Gaudich. & 6 & 1,5 & 1,45 & 0,67 & 3,63 & ST \\
\hline Jacaratia spinosa (Aubl.) A.DC. & 4 & 1 & 1,16 & 1,38 & 3,54 & ST \\
\hline Chrysophyllum flexuosum Mart. & 5 & 1,25 & 1,45 & 0,65 & 3,35 & ST \\
\hline Xylopia laevigata (Mart.) R.E.Fr. & 4 & 1 & 1,16 & 1,18 & 3,34 & ST \\
\hline Trichilia lepidota Mart. & 5 & 1,25 & 0,87 & 1,07 & 3,19 & ST \\
\hline Oxandra nitida R.E.Fr. & 5 & 1,25 & 1,45 & 0,36 & 3,06 & $\mathrm{NC}$ \\
\hline Hirtella angustifolia Schott ex Spreng. & 2 & 0,5 & 0,29 & 2,26 & 3,05 & $\mathrm{NC}$ \\
\hline Pouteria ramiflora (Mart.) Radlk. & 2 & 0,5 & 0,58 & 1,92 & 3 & ST \\
\hline Guapira noxia (Netto) Lundell & 5 & 1,25 & 1,16 & 0,47 & 2,88 & SI \\
\hline Carpotroche brasiliensis (Raddi) A Gray & 4 & 1 & 1,16 & 0,7 & 2,86 & SI \\
\hline Trichilia ramalhoi Rizzini & 4 & 1 & 0,87 & 0,87 & 2,74 & ST \\
\hline Basiloxylon brasiliensis (All.) K. Schum. & 4 & 1 & 1,16 & 0,45 & 2,61 & ST \\
\hline Sapindaceae sp.1 & 3 & 0,75 & 0,58 & 1 & 2,33 & $\mathrm{NC}$ \\
\hline Anadenanthera colubrina var. cebil (Griseb.) Altschul & 3 & 0,75 & 0,87 & 0,42 & 2,04 & SI \\
\hline Pouteria sp. & 3 & 0,75 & 0,87 & 0,36 & 1,98 & $\mathrm{NC}$ \\
\hline
\end{tabular}




\begin{tabular}{|c|c|c|c|c|c|c|}
\hline Espécies & NI & DR & FR & DoR & VI & GE \\
\hline Lonchocarpus cultratus (Vell.) A.M.G. Azevedo \& H.C. Lima & 3 & 0,75 & 0,87 & 0,3 & 1,92 & SI \\
\hline Licania kunthiana Hook.f. & 3 & 0,75 & 0,58 & 0,58 & 1,91 & SI \\
\hline Pseudolmedia laevigata Trécul & 3 & 0,75 & 0,87 & 0,28 & 1,9 & SI \\
\hline Alseis pickelii Pilg. \& Schmale & 3 & 0,75 & 0,87 & 0,27 & 1,89 & SI \\
\hline Brosimum guianense (Aubl.) Huber & 3 & 0,75 & 0,87 & 0,27 & 1,89 & SI \\
\hline Ecclinusa ramiflora Mart. & 3 & 0,75 & 0,87 & 0,23 & 1,86 & ST \\
\hline Ixora venulosa Benth. & 3 & 0,75 & 0,87 & 0,2 & 1,82 & ST \\
\hline Abarema conchliacarpos (Gomes) Barneby \& J.W. Grimes & 3 & 0,75 & 0,87 & 0,17 & 1,79 & ST \\
\hline Trichilia clausseni C.DC. & 3 & 0,75 & 0,58 & 0,43 & 1,76 & ST \\
\hline Andradaea floribunda Allemão & 2 & 0,5 & 0,58 & 0,65 & 1,73 & PI \\
\hline Inga edulis Mart. & 1 & 0,25 & 0,29 & 0,99 & 1,53 & SI \\
\hline Actinostemon sp. & 3 & 0,75 & 0,29 & 0,34 & 1,38 & $\mathrm{NC}$ \\
\hline Bauhinia forficata Link. & 2 & 0,5 & 0,58 & 0,28 & 1,36 & SI \\
\hline Cabralea canjerana (Vell.) Mart. & 2 & 0,5 & 0,58 & 0,26 & 1,34 & SI \\
\hline Terminalia mameluco Pickel & 2 & 0,5 & 0,58 & 0,23 & 1,31 & NC \\
\hline Genipa americana $\mathrm{L}$. & 2 & 0,5 & 0,58 & 0,19 & 1,27 & ST \\
\hline Diploon cuspidatum (Hoehne) Cronquist & 2 & 0,5 & 0,58 & 0,19 & 1,27 & ST \\
\hline Chrysophyllum sp. & 2 & 0,5 & 0,58 & 0,18 & 1,26 & $\mathrm{NC}$ \\
\hline Dalbergia nigra (Vell.) AllemãoexBenth. & 2 & 0,5 & 0,58 & 0,16 & 1,25 & SI \\
\hline Annona dolabripetala Raddi & 2 & 0,5 & 0,58 & 0,13 & 1,21 & SI \\
\hline Guarea guidonia $($ L.) Sleumer & 2 & 0,5 & 0,58 & 0,08 & 1,16 & SI \\
\hline Mimosa bimucronata (DC.) Kuntze & 2 & 0,5 & 0,29 & 0,36 & 1,15 & PI \\
\hline Peltophorum dubium (Spreng.) Taub. & 1 & 0,25 & 0,29 & 0,54 & 1,08 & SI \\
\hline Copaifera langsdorffii Desf. & 1 & 0,25 & 0,29 & 0,51 & 1,05 & ST \\
\hline Ampelocera glabra Kuhlm. & 1 & 0,25 & 0,29 & 0,5 & 1,04 & SI \\
\hline Cecropia sp. & 1 & 0,25 & 0,29 & 0,47 & 1,01 & NC \\
\hline Ficus sp. & 1 & 0,25 & 0,29 & 0,39 & 0,93 & $\mathrm{NC}$ \\
\hline Luehea divaricata Mart. \& Zucc. & 1 & 0,25 & 0,29 & 0,39 & 0,93 & SI \\
\hline Inga hispida Schott ex Benth. & 2 & 0,5 & 0,29 & 0,11 & 0,9 & ST \\
\hline Virola gardneri (A.DC.) Warb. & 1 & 0,25 & 0,29 & 0,29 & 0,83 & ST \\
\hline Sloanea sp.1 & 1 & 0,25 & 0,29 & 0,27 & 0,81 & NC \\
\hline Pouteria caimito (Ruiz \& Pav.) Radlk. & 1 & 0,25 & 0,29 & 0,27 & 0,81 & ST \\
\hline Ziziphus glaziovii Warm. & 1 & 0,25 & 0,29 & 0,27 & 0,81 & ST \\
\hline Celtis sp. & 1 & 0,25 & 0,29 & 0,25 & 0,79 & $\mathrm{NC}$ \\
\hline Guarea sp. & 1 & 0,25 & 0,29 & 0,22 & 0,76 & NC \\
\hline Aspidosperma olivaceum Müll. Arg. & 1 & 0,25 & 0,29 & 0,22 & 0,76 & ST \\
\hline Bauhinia sp.2 & 1 & 0,25 & 0,29 & 0,22 & 0,76 & NC \\
\hline Melanoxylum braúna Schott & 1 & 0,25 & 0,29 & 0,2 & 0,74 & ST \\
\hline Astrocaryum aculeatissimum (Schott.) Burret & 1 & 0,25 & 0,29 & 0,2 & 0,74 & SI \\
\hline Cupania oblongifolia Mart. & 1 & 0,25 & 0,29 & 0,18 & 0,72 & ST \\
\hline
\end{tabular}




\begin{tabular}{|c|c|c|c|c|c|c|}
\hline Espécies & NI & DR & FR & DoR & VI & GE \\
\hline Lauraceae sp.1 & 1 & 0,25 & 0,29 & 0,17 & 0,71 & $\mathrm{NC}$ \\
\hline Maytenus gonoclada Mart. & 1 & 0,25 & 0,29 & 0,14 & 0,68 & SI \\
\hline Matayba sp. & 1 & 0,25 & 0,29 & 0,14 & 0,68 & $\mathrm{NC}$ \\
\hline Guarea sp. & 1 & 0,25 & 0,29 & 0,13 & 0,67 & $\mathrm{NC}$ \\
\hline Chrysophyllum sp. & 1 & 0,25 & 0,29 & 0,12 & 0,66 & $\mathrm{NC}$ \\
\hline Maytenus sp.2 & 1 & 0,25 & 0,29 & 0,11 & 0,65 & $\mathrm{NC}$ \\
\hline Astronium sp. & 1 & 0,25 & 0,29 & 0,11 & 0,65 & $\mathrm{NC}$ \\
\hline Faramea sp. & 1 & 0,25 & 0,29 & 0,1 & 0,64 & NC \\
\hline Ephedranthus sp. & 1 & 0,25 & 0,29 & 0,09 & 0,63 & $\mathrm{NC}$ \\
\hline Protium heptaphyllum (Aubl.) Marchand & 1 & 0,25 & 0,29 & 0,09 & 0,63 & ST \\
\hline Randia armata $(\mathrm{Sw}.) \mathrm{DC}$ & 1 & 0,25 & 0,29 & 0,09 & 0,63 & SI \\
\hline Zanthoxylum rhoifolium Lam. & 1 & 0,25 & 0,29 & 0,08 & 0,62 & PI \\
\hline Cariniana estrellensis (Raddi) Kuntze & 1 & 0,25 & 0,29 & 0,08 & 0,62 & ST \\
\hline Guazuma sp. & 1 & 0,25 & 0,29 & 0,08 & 0,62 & NC \\
\hline Rubiaceae sp.1 & 1 & 0,25 & 0,29 & 0,08 & 0,62 & NC \\
\hline Gallesia integrifolia (Spreng.) Harms & 1 & 0,25 & 0,29 & 0,07 & 0,61 & ST \\
\hline Macrothumia kuhlmannii (Sleumer) M.H. Alford & 1 & 0,25 & 0,29 & 0,07 & 0,61 & ST \\
\hline Chrysophyllum gonocarpum (Mart. \& Eichler ex Miq.) Engl. & 1 & 0,25 & 0,29 & 0,07 & 0,61 & ST \\
\hline Sorocea bonplandii (Baill.) W.C. Burger et al. & 1 & 0,25 & 0,29 & 0,06 & 0,6 & SI \\
\hline Amaioua intermedia Mart. ex Schult. \& Schult.f. & 1 & 0,25 & 0,29 & 0,06 & 0,6 & SI \\
\hline Phyllostylon brasiliense Capan. ex Benth. \& Hook.f. & 1 & 0,25 & 0,29 & 0,06 & 0,6 & $\mathrm{NC}$ \\
\hline Sloanea sp.2 & 1 & 0,25 & 0,29 & 0,06 & 0,6 & $\mathrm{NC}$ \\
\hline Bauhinia sp. 1 & 1 & 0,25 & 0,29 & 0,06 & 0,6 & $\mathrm{NC}$ \\
\hline Matayba juglandifolia (Cambess.) Radlk. & 1 & 0,25 & 0,29 & 0,06 & 0,6 & SI \\
\hline Machaerium brasiliense Vogel & 1 & 0,25 & 0,29 & 0,05 & 0,59 & SI \\
\hline Bathysa cuspidata (A.St.-Hil.) Hook.f. ex K.Schum. & 1 & 0,25 & 0,29 & 0,05 & 0,59 & SI \\
\hline Cupania sp. & 1 & 0,25 & 0,29 & 0,05 & 0,59 & NC \\
\hline Guatteria australis A.St.-Hil. & 1 & 0,25 & 0,29 & 0,05 & 0,59 & SI \\
\hline Moraceae sp.1 & 1 & 0,25 & 0,29 & 0,04 & 0,58 & NC \\
\hline Heisteria silviani Schwacke & 1 & 0,25 & 0,29 & 0,04 & 0,58 & ST \\
\hline Pseudobombax grandiflorum (Cav.) A.Robyns & 1 & 0,25 & 0,29 & 0,04 & 0,58 & SI \\
\hline Couratari macrosperma A.C.Sm. & 1 & 0,25 & 0,29 & 0,04 & 0,58 & ST \\
\hline Pradosia lactescens (Vell.) Radlk. & 1 & 0,25 & 0,29 & 0,03 & 0,57 & ST \\
\hline Annona sylvatica A.St.-Hil. & 1 & 0,25 & 0,29 & 0,03 & 0,57 & SI \\
\hline Maytenus sp.1 & 1 & 0,25 & 0,29 & 0,03 & 0,57 & NC \\
\hline Trichilia sp. & 1 & 0,25 & 0,29 & 0,03 & 0,57 & $\mathrm{NC}$ \\
\hline Eugenia oblongata $\mathrm{O}$. Berg & 1 & 0,25 & 0,29 & 0,03 & 0,57 & ST \\
\hline Família indeterminada - Indet. 1 & 2 & 0,5 & 0,58 & 0,62 & 1,71 & NC \\
\hline Total & 399 & 100 & 100 & 100 & 100 & - \\
\hline
\end{tabular}


As espécies de maior VI variaram entre os trabalhos realizados em remanescentes florestais pertecentes a mesma fitofisionomia da área de estudo, indicando que processos de sucessão, fatores bióticos e abióticos, competição por recursos ou mesmo diferentes critérios de amostragem utilizados influenciam a distribuição e densidade de espécies. Esse fato foi verificado por Abreu et al. (2013) na Floresta Nacional de Pacotuba (FLONA), no qual $P$. contorta não mostrou expressão como na amostragem da ARIE, enquanto no estudo de Archanjo et al. (2012) realizado na
Reserva Particular Patrimônio Natural (RPPN) de Cafundó, $P$. contorta figurou como a segunda mais importante. Além dessas observações, a espécie em questão é relatada em outros estudos fitossociológicos para florestas estacionais semideciduais como sendo uma das principais constituintes dessa fitofisionomia (Santos et al. 2012; Abreu et al. 2014).

A área basal $\left(6,17 \mathrm{~m}^{2} \mathrm{ha}^{-1}\right)$ foi considerada baixa quando comparada a outros estudos na mesma fitofisionomia (Tab. 2). Essa característica está relacionada com a presença de árvores mais

Tabela 2 - CI. critério de inclusão; Nsp. número de espécies; MA. método de amostragem; AAQ. área amostral/ quadrantes; DA. densidade absoluta; AB. área basal; H'. índice de Shannon; J. equabilidade de Pielou.

Table 2 - CI. inclusion criteria; Nsp. number of species; MA. sampling method; AAQ. sample area/quarters; DA. absolute density; AB. basal area; H'. Shannon index; J. Pielou evenness.

\begin{tabular}{|c|c|c|c|c|c|c|c|c|c|}
\hline Local & Nsp & $\begin{array}{c}\text { CI } \\
(\mathrm{cm})\end{array}$ & MA & AAQ & $\begin{array}{c}\text { DA } \\
\left(\text { indha }^{-1}\right)\end{array}$ & $\begin{array}{c}\mathbf{A B} \\
\left(\mathbf{m}^{2} \mathbf{h} \mathbf{a}^{-1}\right)\end{array}$ & $\begin{array}{c}\mathbf{H}^{\prime} \\
\left.\text { (natsind }^{-1}\right)\end{array}$ & $\mathbf{J}$ & Autores \\
\hline $\begin{array}{l}\text { RPPN Cafundó, } \\
\text { Cachoeiro de } \\
\text { Itapemirim, ES }\end{array}$ & 253 & 5 & Parcela & 2,4 ha & 1.488 & 25,72 & 3,31 & 0,60 & $\begin{array}{l}\text { Abreu et al., } \\
\quad 2013\end{array}$ \\
\hline $\begin{array}{l}\text { Flona de Pacotuba, } \\
\text { Cachoeiro de } \\
\text { Itapemirim, ES }\end{array}$ & 255 & 5 & Parcela & $2,5 \mathrm{ha}$ & 1.823 & 33,02 & 4,13 & 0,74 & $\begin{array}{c}\text { Archanjo et al., } \\
2012\end{array}$ \\
\hline Coqueiral, MG & 144 & 5 & Parcela & 0,8 ha & $1.098,75$ & 23,76 & 4,31 & 0,87 & $\begin{array}{c}\text { Moreira et al., } \\
\quad 2013\end{array}$ \\
\hline $\begin{array}{l}\text { Pico do Jabre, } \\
\text { Maturéia e Mãe } \\
\text { d'Água, PB }\end{array}$ & 63 & 4,8 & Parcela & $1,8 \mathrm{ha}$ & 1.138 & 22,45 & 3,17 & 0,76 & $\begin{array}{l}\text { Cunha et al., } \\
2013\end{array}$ \\
\hline $\begin{array}{l}\text { Itambé do Mato } \\
\text { Dentro, MG }\end{array}$ & 115 & 4,8 & $\begin{array}{c}\text { Ponto } \\
\text { Quadrante }\end{array}$ & 100 & 3.638 & - & 4,32 & 0,91 & $\begin{array}{l}\text { Santos et al., } \\
\quad 2012\end{array}$ \\
\hline $\begin{array}{l}\text { Campolina, } \\
\text { Marliérea, MG }\end{array}$ & 90 & 4,8 & $\begin{array}{c}\text { Ponto } \\
\text { Quadrante }\end{array}$ & 70 & $1.325,72$ & 36,41 & 4,1 & 0,91 & $\begin{array}{c}\text { França e } \\
\text { Stehmann, } 2013\end{array}$ \\
\hline $\begin{array}{l}\text { Macuco, Timóteo, } \\
\text { MG }\end{array}$ & 100 & 4,8 & $\begin{array}{c}\text { Ponto } \\
\text { Quadrante }\end{array}$ & 70 & $1.427,45$ & 20,9 & 4,07 & 0,89 & $\begin{array}{c}\text { França e } \\
\text { Stehmann, } 2013\end{array}$ \\
\hline $\begin{array}{l}\text { Mumbaça, } \\
\text { Dionísio, MG }\end{array}$ & 96 & 4,8 & $\begin{array}{c}\text { Ponto } \\
\text { Quadrante }\end{array}$ & 70 & $1.366,36$ & 22,16 & 3,99 & 0,88 & $\begin{array}{c}\text { França e } \\
\text { Stehmann, } 2013\end{array}$ \\
\hline $\begin{array}{l}\text { Fazenda } \\
\text { Macedônia, Ipaba, } \\
\text { MG }\end{array}$ & 93 & 4,8 & $\begin{array}{c}\text { Ponto } \\
\text { Quadrante }\end{array}$ & 70 & $1.597,65$ & 20,9 & 3,95 & 0,87 & $\begin{array}{c}\text { França e } \\
\text { Stehmann, } 2013\end{array}$ \\
\hline $\begin{array}{l}\text { Fazenda } \\
\text { Sacramento, Pingo } \\
\text { d'Água, MG }\end{array}$ & 101 & 4,8 & $\begin{array}{c}\text { Ponto } \\
\text { Quadrante }\end{array}$ & 70 & $1.769,14$ & 39,48 & 3,94 & 0,86 & $\begin{array}{c}\text { França e } \\
\text { Stehmann, } 2013\end{array}$ \\
\hline $\begin{array}{l}\text { Morro do Gavião, } \\
\text { Dionísio, MG }\end{array}$ & 80 & 4,8 & $\begin{array}{c}\text { Ponto } \\
\text { Quadrante }\end{array}$ & 70 & $1.364,15$ & 27,07 & 3,66 & 0,84 & $\begin{array}{c}\text { França e } \\
\text { Stehmann, } 2013\end{array}$ \\
\hline $\begin{array}{l}\text { ARIE Laerth Paiva } \\
\text { Gama, Alegre, ES }\end{array}$ & 112 & 5 & $\begin{array}{c}\text { Ponto } \\
\text { Quadrante }\end{array}$ & 100 & $2.046,34$ & 6,2 & 4,17 & 0,87 & Este estudo \\
\hline
\end{tabular}


esparsas em alguns trechos, indicando a existência de áreas com influência de borda, clareiras e encostas com intenso processo dinâmico.

Já a diversidade florística estimada pelo índice de Shannon (H') apresentou elevado valor $\left(4,17\right.$ natsind $\left.^{-1}\right)$ em resposta à diversidade e abundância de espécies, não ocorrendo domínio de uma determinada entidade. Esses valores estão acima dos comumente encontrados para florestas estacionais semideciduais como resposta a uma série de fatores, principalmente os relacionados ao estágio sucessional da floresta, já que ambientes perturbados apresentam maior riqueza de espécies que florestas maduras (Gris et al. 2014). A equabilidade $(\mathrm{J})$ foi de 0,87 , indicando que $87 \%$ da diversidade máxima teórica foi representada na amostragem, além da uniformidade na distribuição dos indivíduos entre as espécies (Tab. 2). Essas estimativas mostram a importância da Unidade de Conservação estudada para a manutenção da diversidade florística, mesmo se tratando de um pequeno fragmento florestal e estando sob elevada pressão antrópica.

A análise de grupos ecológicos revelou maior número de espécies secundárias iniciais $(33,92 \%)$ e tardias $(33,92 \%)$, representando aproximadamente $68 \%$ do total de espécies amostradas na vegetação estudada. Comportamento similar foi observado ao analisar a distribuição dos indivíduos nas classes ecológicas, em que $74,4 \%$ do total de indivíduos inventariados compuseram os grupos de secundárias iniciais (155 indivíduos) e tardias (142).

Entretanto, desconsiderando aqueles indivíduos que não tiveram classificação, seja por não estarem em nível de espécie ou não encontrados na literatura, o número de indivíduos pertencentes a espécies pioneiras e secundárias iniciais corresponderam a mais da metade dos indivíduos relacionadas na amostragem (Tab. 3). Esses dois grupos são os responsáveis diretos pela colonização inicial de florestas perturbadas, podendo conceder um indicativo de que a vegetação estudada está se conduzindo para a fase intermediária de sucessão. Este fato pode ser sustentado levando em consideração que das cinco espécies de maior VI, três pertencem ao grupo ecológico secundária inicial e uma à pioneira.

A presença destacada de espécies consideradas como ruderais pode ser um indicativo da má qualidade de fragmentos, neste caso verifica-se a ocorrência de $P$. contorta entre as espécies de maior VI na área amostrada corroborando com os estudos em outros fragmentos caracterizados como perturbados (Peixoto et al. 2005; Dan et al. 2010). Segundo Alves \& Metzger (2006), o enriquecimento e o avanço sucessional das florestas secundárias dependem da chegada de propágulos das espécies finais de sucessão.

Apesar dessas observações, é importante mencionar que as espécies pioneiras e secundárias iniciais contribuíram com mais da metade da área basal calculada (Tab. 3) para a vegetação $(67,42 \%$ ou $4,16 \mathrm{~m}^{2} \cdot \mathrm{ha}^{-1}$ ), indicando também a importância estrutural desses dois grupos ecológicos no contexto de sucessão da floresta.

A avaliação dos grupos ecológicos pode auxiliar na caracterização sucessional da floresta (Gandolfi et al. 1995). No entanto, segundo Silva et al. (2003), a classificação das espécies em grupos ecológicos tem sido imprecisa em relação a dois fatores primordiais. O primeiro refere-se

Tabela 3 - Representatividade dos grupos ecológicos das espécies encontradas na ARIE Laerth Paiva Gama, Alegre, ES - GE. grupos ecológicos; Nsp. número de espécies; PN. percentual de espécies; n. número de indivíduos; PI. percentual de indivíduos; PI. pioneira; SI. secundária inicial; ST. secundária tardia; NC. não caracterizada.

Table 3 - Representation of ecological groups of species founded in the ARIE Laerth Paiva Gama, Alegre, Espirito Santo - GE. ecological groups; Nsp. number of species; PN. percentage of species; n. number of individuals; PN. percentage of individuals; PI. pioneer; SI. Initial secondary; ST. late secondary; NC. not characterized.

\begin{tabular}{ccccccc}
\hline GE & Nsp & PN (\%) & $\mathbf{n}$ & Pn (\%) & $\mathbf{A b ~ ( \mathbf { m } ^ { 2 } )}$ & $\mathbf{A b} \mathbf{( \% )}$ \\
\hline PI & 5 & 4,46 & 57 & 14,28 & 1,79 & 29,01 \\
SI & 38 & 33,92 & 155 & 38,84 & 2,37 & 38,41 \\
ST & 38 & 33,92 & 142 & 35,58 & 1,48 & 23,99 \\
NC & 31 & 27,67 & 45 & 11,27 & 0,53 & 8,59 \\
Total & $\mathbf{1 1 2}$ & $\mathbf{1 0 0}$ & $\mathbf{3 9 9}$ & $\mathbf{1 0 0}$ & $\mathbf{6 , 1 7}$ & $\mathbf{1 0 0}$ \\
\hline
\end{tabular}


aos critérios utilizados entre diferentes autores, conduzindo a distintas formas de ordenação das espécies. A segunda razão assinalada seria a respeito da resposta de uma determinada espécie em relação a ambientes com disponibilidades distintas de recursos.

As florestas são comunidades vegetais dinâmicas e processos sucessionais são intrínsecos desses sistemas. As variações na dinâmica florestal influenciam a estrutura, composição florística e mecanismos de dispersão (Nascimento et al. 2014), por isso, essas e outras variáveis como a regeneração natural, estrutura diamétrica e área basal também devem ser consideradas para definição do estágio sucessional de um remanescente florestal.

Assim, a distribuição diamétrica (Fig. 2) para a vegetação estudada não seguiu o padrão típico, para florestas naturais, de $J$ invertido (Soares et al. 2006), apesar do direcionamento a essa tendência. A primeira classe de diâmetro incluiu aproximadamente 55\% dos indivíduos amostrados, do mesmo modo a segunda e a terceira classe apresentaram percentual expressivo de indivíduos $(34,5 \%)$, proporcionando a estrutura diamétrica fora do esperado para florestas inequiâneas.

Esse padrão pode estar relacionado ao estágio sucessional geral da vegetação (Soares et al. 2006), em que florestas em estágio inicial e mais perturbadas apresentam número relevante de indivíduos nas classes intermediárias e finais, enquanto florestas maduras exibem estrutura diamétrica caracterizada pela concentração de indivíduos na primeira classe, fornecendo indícios de que a regeneração está seguindo de maneira aparentemente adequada. Outra questão estaria associada a não uniformidade de características ecológicas e ambientais da própria vegetação avaliada por apresentarem estágios sucessionais

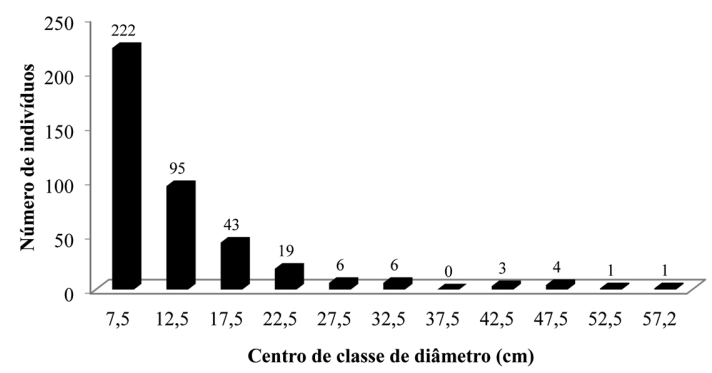

Figura 2 - Distribuição diamétrica dos indivíduos amostrados na ARIE Laerth Paiva Gama, Alegre, ES. Figure 2 - Distribution of diametric individuals sampled in ARIE Laerth Paiva Gama, Alegre, Espirito Santo. diferentes uns dos outros em várias porções na floresta, dependendo das alterações sofridas tanto por intervenções antrópicas como processos inerentes da dinâmica florestal e influência de bordas. Essas mudanças podem estar também relacionadas às interações interespecíficas, inclusive competição e ordem de chegada de espécies colonizadoras (Walker et al. 2010), mecanismos ainda pouco estudados nesse contexto.

Dessa forma, a avaliação da estrutura diamétrica agrega informação para inferir sobre as condições da vegetação, auxiliando a definição de parâmetros para tomadas de decisões em diversas atividades como plano de manejo florestal, intervenções para conduzir a sucessão ecológica e licenciamento ambiental.

A distribuição diamétrica para as cinco espécies de maior VI (Fig. 3), seguiu padrões variáveis. As espécies de grupos ecológicos iniciais apresentaram distribuição de seus indivíduos em todas as classes de diâmetro, sendo relevante, inclusive, em classes maiores, exceto $A$. leiocarpa. Em contrapartida, a espécie de grupo sucessional mais avançado (A. lentiscifolium) teve tendência ao $J$ invertido.

Esses resultados mostram que a capacidade da avaliação em nível de espécies da população pode auxiliar na inferência sobre quais espécies estão em declínio na comunidade vegetal e quais estão se estabelecendo no ambiente. Em outras palavras, a distribuição diamétrica por espécies permite avaliar a substituição das espécies ao longo do tempo, ou seja, como os processos de sucessão ecológica estão atuando sobre elas.

Algumas das espécies merecem atenção especial, principalmente sob a tutela conservacionista, pois estão inseridas no livro vermelho da Flora do Brasil. Apuleia leiocarpa, Dalbergia nigra e Melanoxylon brauna tiveram suas populações reduzidas ao longo dos anos sob intensa exploração madeireira e expansão agrícola, mesmo assim suas distribuições permanecem amplas no Brasil, desta forma, elas foram classificadas como vulneráveis (Lima et al. 2013). Apesar disso, a população de A. leiocarpa (16 indivíduos), na área de estudo, se destacou por sua boa representatividade, ocupando o segundo maior VI, e por possuir regeneração natural bem estabelecida como verificada anteriormente na distribuição diamétrica. Entretanto, D. nigra (dois indivíduos) e $M$. brauna (um indivíduo) foram menos representativas em termos de densidade e dominância, apresentando principalmente indivíduos jovens. Esse comportamento pode ser 

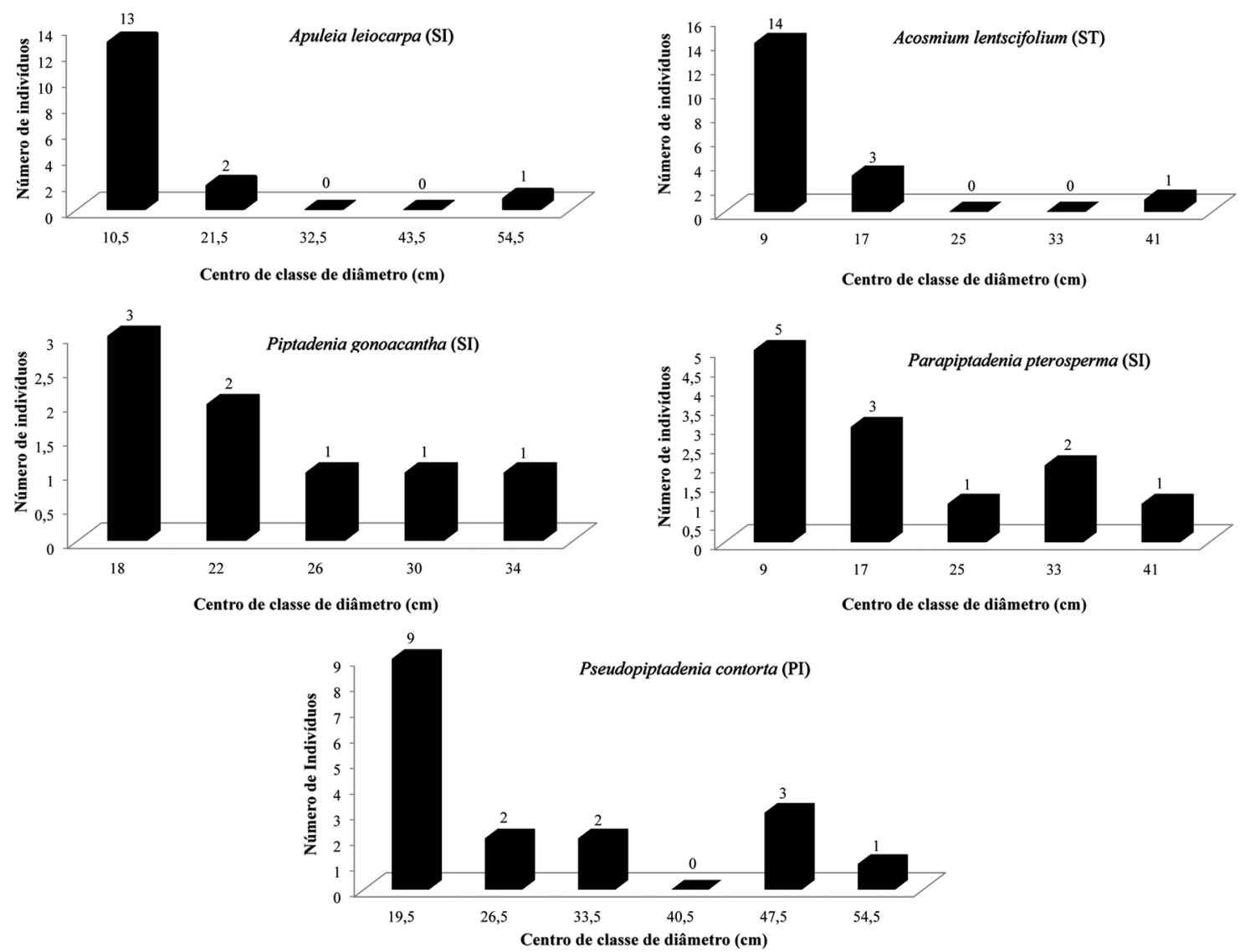

Figura 3 - Distribuição diamétrica dos cinco indivíduos com maior valor de importância na ARIE Laerth Paiva Gama, Alegre, ES. PI = pioneira; SI = secundária inicial; ST = secundária tardia.

Figure 3 - Distribution of diametric of five individuals with the highest importance value in ARIE Laerth Paiva Gama, Alegre, Espirito Santo. PI = pioneer; $\mathrm{SI}=$ early secondary; ST = late secondary.

explicado por meio das estratégias ecológicas de cada espécie, em que $M$. brauna por ser secundária tardia está começando a se estabelecer no local após encontrar condição propícia, ao passo que, D. nigra por ser espécie de estágios sucessionais iniciais, aparenta ter se estabelecido recentemente na vegetação. Essas verificações reforçam a importância do remanescente florestal estudado, além de ampliar o apelo para conservação dessas espécies que podem estar com variabilidade genética reduzida devido ao isolamento de suas populações.

Diante das avaliações apresentadas, têm-se o seguinte problema relacionado aos processos ecológicos na vegetação estudada: reduzida área basal e estrutura diamétrica não bem estabelecida frente às elevadas diversidade florística e densidade de indivíduos em relação a muitas florestas semideciduais já estudadas. Esses inconvenientes estão associados aos mecanismos de distúrbio inerentes à história de ocupação do entorno e à extração seletiva de madeira no passado. Entretanto, diversas porções da vegetação parecem não conseguir se estabilizarem naturalmente, principalmente na borda da comunidade florestal onde os processos são mais intensos. De maneira geral, o remanescente florestal estudado pode ser caracterizado como pertencente ao estágio sucessional inicial-intermediário.

\section{Considerações finais}

A partir da apresentação dos resultados, observa-se que a estrutura fitossociológica da floresta nativa da ARIE Laerth Paiva Gama não encontra-se bem estabelecida ou similar a florestas estacionais mais conservadas, sugerindo que intervenções devem ser aplicadas no sentido de melhorar essas características. 
Arepresentatividade de espécies caracterizadas como pioneiras e secundárias iniciais na comunidade indica que o fragmento florestal pode estar em desenvolvimento para a fase intermediária (estágio inicial-intermediário), embora sugere-se inclusão de novos parâmetros de avaliação dos estágios sucessionais em remanescentes.

Implementa-se com esse estudo, a melhoria da eficiência na gestão e manejo da Unidade de Conservação pesquisada podendo ser direcionada ao fortalecimento das atividades de Educação Ambiental e levantamento de propostas para conexão de fragmentos florestais próximos à ARIE como forma de proteger a diversidade e a manutenção da dinâmica florestal. Além disso, é importante a utilização desses dados no levantamento de propostas e estratégias para conduzir o referido remanescente a uma condição avançada de sucessão.

\section{Agradecimentos}

Agradecemos ao Senhor Ademar Emídio da Silva, o auxílio nas coletas de materiais botânicos; e à Pesquisadora do Instituto Florestal do estado de São Paulo, Natália Ivanauskas, as contribuições realizadas na determinação das espécies de plantas.

\section{Referências}

Abreu, K.M.P.; Braga, J.M.A. \& Nascimento, M.T. 2014. Tree species diversity of Coastal Lowland Semideciduous Forest fragments in northern Rio de Janeiro State, Brazil. Bioscience Journal 30: 1529-1550.

Abreu, K.M.P.; Silva, G.F. \& Silva, A.G. 2013. Análise fitossociológica da Floresta Nacional de Pacotuba, Cachoeiro de Itapemirim, ES - Brasil. Cerne 19: 157-168.

Almeida, C.M. de; Araujo, M.M.; Longhi, S.J.; Rovedder, A.P.; Sccoti, M.S.V.; d'Avila, M.; Aimi, S.C. \& Tonetto, T.S. 2015. Análise de agrupamentos em remanescente de Floresta Estacional Decidual. Ciência Florestal 25: 781-789.

Alvares, C.A.; Stape, J.L.; Sentelhas, P.C.; Gonçalves, J.L.M. \& Sparovek, G. 2013. Köppen's climate classification map for Brazil. Meteorologische Zeitschrift 22: 711-728.

Alves, L.F. \& Metzger, J.P. 2006. A regeneração florestal em áreas de floresta secundária na Reserva Florestal do Morro Grande, Cotia, SP. Biota Neotropica 6: 1-26.

Angiosperm Phylogeny Group (APG). 2016. An update of the Angiosperm Phylogeny Group classification for the orders and families of flowering plants: APGIV. Botanical Journal of the Linnean Society 181: 1-20.

Araújo, E.A.; Moreira, G.L.; Santos, J.P.Q.; Zorzanelli, J.P.F. \& Kunz, S.H. 2015. Dinâmica da cobertura vegetaldo corredor ecológico Burarama-PacotubaCafundó, ES. In: Santos, A.R.; Ribeiro, C.A.A.S.; Peluzio, J.B.E.; Santos, G.M.A.D.A.; Moreira, G.L. \& Magalhães, I.A.L. (orgs.). Geotecnologias e análise ambiental: aplicações práticas. CAUFES, Alegre. Pp. 118-133.

Archanjo, K.M.P.A.; Silva, G.F.; Chichorro, J.F. \& Soares, C.P.B. 2012. Estrutura do componente arbóreo da Reserva Particular do Patrimônio Natural Cafundó, Cachoeiro de Itapemrim, Espírito Santo, Brasil. Floresta 42: 145-160.

Bianchini, E.; Garcia, C.C.; Pimenta, J.A. \& Torezan, J.M.D. 2010. Slope variation and population structure of tree species from different ecological groups in South Brazil. Anais da Academia Brasileira de Ciências 82: 643-52.

Braga, A.J.T.; Borges, E.E.L. \& Martins, S.V. 2011. Florística e estrutura da comunidade arbórea de uma Floresta Estacional Semidecidual secundária em Viçosa, MG. Revista Árvore 35: 493-503.

Brower, J.E.; Zar, J.H. \& Von Ende, C.N. 1998. Field and laboratory methods for general ecology. $4^{\text {th }}$ ed. McGraw-Hill, New York. 288p.

Budowski, G. 1965. Distribution of tropical american rain forest species in the light of sucessional processes. Turrialba 15: 40-42.

Carvalho, F.A.; Nascimento, M.T. \& Braga, J.M.A. 2007. Estrutura e composição florística do estrato arbóreo de um remanescente de Mata Atlântica Submontana no município de Rio Bonito, RJ, Brasil (Mata Rio Vermelho). Revista Árvore 31: 717-730.

Colombo, A.F. \& Joly, C.A. 2010. Brazilian Atlantic Forest lato sensu: the most ancient Brazilian forest, and a biodiversity hotspot, is highly threatened by climate change. Brazilian Journal of Biology 70: 697-708.

Dan, M.L.; Braga, J.M.A. \& Nascimento, M.T. 2010. Estrutura da comunidade arbórea de fragmentos de floresta estacional semidecidual na Bacia Hidrográfica do Rio São Domingos, São José de Ubá, Rio de Janeiro, Brasil. Rodriguésia 61: 749-766.

Durigan, G. 2003. Métodos para análise de vegetação arbórea. In: Cullen Junior, L.; Rudran, R. \& Valladares-Pádua, C. (orgs.). Métodos de estudos em biologia da conservação e manejo da vida silvestre. UFPR. Fundação Boticário de Proteção à Natureza, Curitiba. Pp. 455-479.

Espírito Santo. 1994. Secretaria de Estado de Ações Estratégicas e Planejamento. Departamento Estadual de Estatística. Informações municipais do estado do Espírito Santo. Vitória 1: 803.

Flora do Brasil 2020 [em construção]. 2016. Jardim Botânico do Rio de Janeiro. Disponível em <http:// floradobrasil.jbrj.gov.br>. Acesso em 13 junho 2016.

Fonseca, R.C.B. \& Rodrigues, R.R. 2000. Análise estrutural e aspectos do mosaico sucessional de uma floresta semidecídua em Botucatu, SP. Scientia Forestalis 57: 27-43. 
Forzza, R.C.; Baumgratz, J.F.A.; Bicudo, C.E.M.; Canhos, D.A.L.; Carvalho Junior, A.A.; Coelho, M.A.N.; Costa, A.F.; Costa, D.P.; Hopkins, M.G.; Leitman, P.M.; Lohmann, L.G.; Lughadha, E.N.; Maia, L.C.; Martinelli, G.; Menezes, M.; Morim, M.P.; Peixoto, A.L.; Pirani, J.R.; Prado, J.; Queiroz, L.P.; Souza, S.; Souza, V.C.; Stehmann, J.R.; Sylvestre, L.S.;Walter, B.M.T. \& Zappi, D.C. 2012. New brazilian floristic list highlights conservation challenges. Biosciense 62: 39-45.

Gandolfi, S.; Leitão-Filho, H.F. \& Bezerra, C.L.F. 1995. Levantamento florístico e caráter sucessional das espécies arbustivo-arbóreas de uma Floresta Mesófila Semidecídua no município de Guarulhos, SP. Revista Brasileira de Biologia 55: 753-767.

Gris, D.; Temponi, L.G. \& Damasceno-Junior, G.A. 2014. Structure and floristic diversity of remnant Semideciduous Forest under varying levels of disturbance. Acta Botanica Brasilica 28: 569-576.

Imaña-Encinas, J.; Santana, O.A.; Macedo, L.A. \& Paula, J.E. 2008. Distribuição diamétrica de um trecho da Floresta Estacional Semidecidual na área do Ecomuseu do Cerrado. Cerne 14: 33-45.

Joly, C.A.; Metzger, J.P. \& Tabarelli, M. 2014. Experiences from the Brazilian Atlantic Forest: ecological findings and conservation initiatives. New Phytologist 204: 459-473.

Lima, H.C.; Souza, E.R.; Tozzi, A.M.G.A.; Perez, A.P.F.; Flores, A.S.; Sartori, A.L.B.; Vaz, A.M.S.F.; Fernandes, F.F.F.M.; Garcia, F.C.P.; Iganci, J.R.V.; Fernandes, J.M.; Valls, J.F.M.; Lima, L.C.P.; Costa, L.C.; Queiroz, L.P.; Morim, M.P.; Nunes, N.L.A.; Queiroz, R.T.; Miotto, S.T.S.; Dutra, V.F.; Mansano, V.F.; Souza, V.C.; Messina, T.; Kutschenko, D.C.; Penedo, T.S.A. \& Valente, A.M. 2013. Fabaceae/ Leguminosae. In: Martinelli, G. \& Moraes, M.A. (orgs.). Livro Vermelho da Flora do Brasil. Instituto de Pesquisas Jardim Botânico do Rio de Janeiro \& Andrea Jakobson, Rio de Janeiro. Pp. 516-548.

Lopes, S.F.; Schiavini, I.; Prado Júnior, J.A.; Gusson, A.E.; Souza Neto, A.R.; Vale, V.S. \& Dias Neto, O.C. 2011. Caracterização ecológica e distribuição diamétrica da vegetação arbórea em um remanescente de Floresta Estacional Semidecidual, na Fazenda Experimental do Glória, Uberlândia, MG. Bioscience Journal 27: 322-335.

Marangon, L.C.; Soares, J.J.; Feliciano, A.L.P. \& Brandão, C.F.LeS. 2007. Estrutura fitossociológica e classificação sucessional do componente arbóreo de um fragmento de floresta estacional semidecidual, no município de Viçosa, Minas Gerais. Cerne 13: 208-221.

Martinelli, G. \& Moraes, M.A. 2013. Livro Vermelho da Flora do Brasil. Instituto de Pesquisas Jardim Botânico do Rio de Janeiro \& Andrea Jakobson, Rio de Janeiro. 1100p.

Matte, A.L.L.; Müller, S.C. \& Becker, F.G. 2015. Forest expansion or fragmentation? Discriminating forest fragments from natural forest patches through patch structure and spatial context metrics. Austral Ecology 41: 21-31.

Metzger, J.P.; Martensen, A.C.; Dixo, M.; Bernacci, L.C.; Ribeiro, M.C.; Teixeira, A.M.G. \& Pardini, R. 2009. Time-lag in biological responses to landscape changes in a highly dynamic Atlantic forest region. Conservation Biology 142: 1166-1177.

Moreira, A.M.; Menino, G.C.O.; Santos, R.M.; Pifano, D.S.; Borém, R.A.T.; Almeida, C.A.M. \& Domingos, D.Q. 2013. Composição florística e estrutura da comunidade arbórea de um fragmento de Floresta Estacional Semidecidual em Coqueiral, MG, Brasil. Revista Brasileira de Biociências 11: 43-51.

Myers, N.; Mittermeier, R.A.; Mittermeier, C.G.; Fonseca, G.A.B. \& Kent, J. 2000. Biodiversity hotspots for conservation priorities. Nature 403: 853-858.

Nascimento, L.M.; Sampaio, E.V.S.B.; Rodal, M.J.N. \& Lins-e-Silva, A.C.B. 2014. Secondary succession in a fragmented Atlantic Forest landscape: evidence of structural and diversity convergence along a chronosequence. Journal of Forest Research 19: 501-513.

Pardini, R.; Faria, D.; Accacio, G.M.; Laps, R.R.; Mariano-Neto, E.; Paciencia, M.L.B.; Dixo, M. \& Baumgarten, J. 2009. The challenge of maintaining Atlantic forest biodiversity: A multitaxa conservation assessment of specialist and generalist species in an agro-forestry mosaic in southern Bahia. Biological Conservation 142: 1178-1190.

Peixoto, G.L.; Martins, S.V.; Silva, A.F. \& Silva, E. 2005. Composição florística do componente arbóreo de um trecho de Floresta Atlântica na Área de Proteção Ambiental da Serra da Capoeira Grande, Rio de Janeiro, RJ. Acta Botanica Brasilica 18: 151-160.

Pennington, R.T.; Lavin, M. \& Oliveira-Filho, A.T. 2009. Woody plant diversity, evolution, and ecology in the tropics: perspectives from Seasonally Dry Tropical Forests. Annual Review of Ecology, Evolution and Systematics 40: 437-457.

Pereira, J.A.A.; Oliveira-Filho, A.T.; Eisenlohr, P.V.; Miranda, P.L.S. \& Lemos-Filho, J.P. 2015. Human impacts affect tree community features of 20 forest fragments of a vanishing neotropical hotspot. Environmental Management 55: 296-307.

Prach, K. \& Walker, L.R. 2011. Four opportunities for studies of ecological sucession. Trends in Ecology and Evolution 26: 119-123.

Rezende, V.L.; Miranda, P.L.S.; Meyer, L.; Moreira, C.V.; Linhares, M.F.M.; Oliveira-Filho, A.T. \& Eisenlohr, P.V. 2015. Tree species composition and richness along altitudinal gradients as a tool for conservation decisions: the case of Atlantic Semideciduous Forest. Biodiversity and Conservation 24: 2149-2163. 
Ribeiro, M.C.; Metzger, J.P.; Martensen, A.C.; Ponzoni, F.J. \& Hirota, M.M. 2009. The Brazilian Atlantic Forest: how much is left, and how is the remaining forest distributed? Implications for conservation. Biological Conservation 142: 1141-1153.

Rodrigues, P.J.F.P. \& Nascimento, M.T. 2006. Fragmentação florestal: breves considerações teóricas sobre efeitos de borda. Rodriguésia 57: 63-74.

Santos, J.H.S.; Ferreira, R.L.C.; Silva, J.A.A.; Souza, A.L.S.; Santos, E.S. \& Meunier, I.M.J. 2004. Distinção de grupos ecológicos de espécies florestais por meio de técnicas multivariadas. Revista Árvore 28: 387-396.

Santos, M.F.; Serafim, H. \& Sano, P.T. 2012. Composição e estrutura arbórea em Floresta Estacional Semidecidual no Espinhaço Meridional (Serra do Cipó, MG). Rodriguésia 63: 985-997.

Scarano, F.R. \& Ceotto, P. 2015. Brazilian Atlantic Forest: impact, vulnerability, and adaptation to climate change. Biodiversity and Conservation 24: 2319-2331.

Sevilha, A.C.; Paula, A.; Lopes, W.P. \& Silva, A.F. 2001. Fitossociologia do estrato arbóreo de um trecho de floresta estacional no Jardim Botânico da Universidade Federal de Viçosa (Face Sudoeste), Viçosa, Minas Gerais. Revista Árvore 25: 431-443.

Shepherd, G.J. 2009. Fitopac 2.01 - Manual do usuário. UNICAMP, Campinas. 90p.

Silva, A.F. da; Oliveira, R.V. de; Santos, N.R.L. \& Paula, A. de. 2003. Composição florística e grupos ecológicos das espécies de um trecho de Floresta Semidecídua Submontana da Fazenda São Geraldo, Viçosa-MG. Revista Árvore 27: 311-319.

Silva, C.T. da; Reis, G.G. dos; Reis, M.G.F.; Silva, E. \& Chaves, R.A. 2004. Avaliação temporal da florística arbórea de uma floresta secundária no município de Viçosa, Minas Gerais. Revista Árvore 28: 429-441.

Soares, C.P.B.; Paula Neto, F. de \& Souza, A.L. de. 2006. Dendrometria e inventário florestal. UFV, Viçosa. 276p.

Souza, P.B.; Souza, A.L. \& Meira-Neto, J.A.A. 2012. Estrutura diamétrica dos estratos e grupos ecológicos de uma área de floresta estacional semidecidual, em Dionísio, MG. Revista Árvore 36: 151-160.

Souza, P.B.; Meira-neto, J.A.A. \& Souza, A.L. 2013. Diversidade florística e estrutura fitossociológica de um gradiente topográfico em Floresta Estacional Semidecidual Submontana, MG. Cerne 19: 489-499.

Spiegel, M.R. 1976. Estatística. McGraw-Hill, São Paulo. 580p.

Tabarelli, M.; Aguiar, A.V.; Ribeiro, M.C.; Metzger, J.P. \& Peres, C.A. 2010. Prospects for biodiversity conservation in the Atlantic Forest: Lessons from aging human-modified landscapes. Biological Conservation 143: 2328-2340.

Walker, L.R.; Landau, F.H.; Velázquez, E.; Shiels, A.B. \& Sparrow, A.D. 2010. Early successional wood plants facilitate and ferns inhibit forest development on Puerto Rican landslides. Journal of Ecology 98: 625-635. 